\title{
Breast abscess due to Salmonella paratyphi A : Case reports with review of literature
}

\author{
Sonu Agrawal ${ }^{1}$, Vishwanath Singh Yadav $^{1}$, Anurag Srivastava ${ }^{2}$, Arti Kapil $^{1}$, Benu Dhawan ${ }^{1, *}$ \\ ${ }^{1}$ Department of Microbiology, All India Institute Of Medical Sciences, New Delhi, India; \\ ${ }^{2}$ Department of Surgery, All India Institute Of Medical Sciences, New Delhi, India.
}

\begin{abstract}
Summary Salmonella paratyphi A causes paratyphoid fever which is characterized by acute onset of fever, abdominal pain, diarrhoea, nausea and vomiting. Localized disease can occur following both overt and silent bacteremia followed by seeding of bacteria at distant sites. Salmonella species though associated with abscess formation in various organs,are rarely associated with breast abscess. We report 2 cases of breast abscess due to Salmonella enterica serotype paratyphi A. Appropriate sampling, surgery supplemented by a comprehensive microbiological work up aided in pathogen identification and appropriate antibiotic administration for a successful outcome of these patients.
\end{abstract}

Keywords: Breast absecess, Salmonella species, haematogenous spread, pus, drainage

\section{Introduction}

Salmonellae are gram-negative bacilli and disease caused by Salmonella organisms can be divided into 2 categories: typhoidal and non-typhoidal. The reservoir for typhoidal disease is human, but non-typhoidal salmonellae are widely distributed among animals. In humans, nontyphoidal Salmonella infections are most often associated with food products; the rest is nosocomial infections or is acquired from pets (1). The aetiological agents of breast abscess are diverse, Staphylococcus aureus is the most common cause of breast abscess (2) (Table 1). Salmonella species though associated with abscess formation in various organs, are rarely associated with breast abscess. We report 2 cases of breast abscess due to salmonella enterica serotype paratyphi A. Drainage of the abscess followed by appropriate antibiotic treatment resulted in a good clinical outcome. We also reviewed the literature on this entity.

\section{Case Reports}

Released online in J-STAGE as advance publication May 23, 2018.

*Address correspondence to:

Dr.Benu Dhawan, Department of Microbiology, All India Institute of Medical Sciences, New Delhi-110029, India.

E-mail: dhawanb@gmail.com

\subsection{Case I}

A 27-year-old female presented to the surgical outpatient department of AIIMS hospital in 2017 with complaints of painful lump in her left breast for 2 months duration. The patient's history revealed that the lump had started to grow in size gradually for two months. The lump was initially firm and painless, but gradually grew in size and became painful. The patient was not pregnant or lactating at that time. The patient had no other comorbidities. She was afebrile on admission, and all the vital signs were within normal limits. On local examination, the left breast was tender and swollen. A mobile, soft, fluctuating mass of around $5 \times 6 \mathrm{~cm}$ in size located in the left lower quadrant was palpable. The overlying skin was warm and erythematous. There was no spontaneous discharge from the abscess and no lymphadenopathy. There was no nipple retraction or discharge from the nipple. Axillary lymph nodes were not palpable. Rest of physical and systemic examination was normal. Ultrasonography (USG) of the left breast revealed a heterogeneously hypoechoic deep-seated irregular collection of approximately 4.9 $\times 3.5 \mathrm{~cm}$ size. A diagnosis of breast abscess was made. The abscess was drained by USG guided aspiration and treated empirically with oral amoxicillin-clavulanic acid $625 \mathrm{mg} 8$ hourly. Her laboratory parameter revealed a total leukocyte count of $12,500 / \mathrm{mm}^{3}$, with $70 \%$ polymorphonuclear leukocytes. Pus sample was 
Table 1. Causative Organisms for Breast Abscesses (2)

\author{
Aerobic gram-positive bacteria \\ Staphylococci, Streptococci \\ Aerobic gram-negative bacteria \\ Escherichia coli, Klebsiella pneumoniae, Pseudomonas aeruginosa, Proteus mirabilis, Salmonella spp \\ Anaerobic bacteria \\ Peptostreptococcus, Propionibacterium, Bacteroides, Lactobacillus, Eubacterium, Clostridium,Fusobacterium, Veillonella \\ Fungi \\ Candida, Cryptococcus \\ Other causes \\ Bartonella henselae, Mycobacteria, Actinomyces, Brucella \\ Parasites \\ Maggot infestation
}

collected and sent for microbiological investigations. Ziehl-Neelsen stain did not demonstrate acid fast bacilli (AFB) and GeneXpert was negative for Mycobacterium tuberculosis. Gram stain of pus showed numerous polymorphonuclear leukocytes and Gram-negative bacilli. Pus culture grew cream coloured non-hemolytic colonieson 5\% sheep blood agar and nonlactosefermenting colonies on MacConkey agar which were identified as Salmonella enterica paratyphi A by matrixassisted laser desorption ionization-time of flight mass spectrometry using the bioMérieux VITEK MS system (IVD database version 2.0) (USA). The antibiotic susceptibility was determined by Kirby Bauer disk diffusion method in accordance with CLSI guidelines and the isolate was susceptible to ciprofloxacin, trimethoprim-sulfamethoxazole, erythromycin, azithromycin, chloramphenicol, ceftazidime, ceftriaxone, ampicillin and Nalidixic acid.

The patient was called back to re-evaluate the detailed history. Three months ago she had gastroenteritis which resolved without antimicrobial treatment. Stool, urine, and blood samples were obtained for culture and found negative for Salmonella paratyphi A spp. Widal test was also found negative. Subsequently she was treated with tablet azithromycin $1000 \mathrm{mg}$ once daily for five days. The patient responded well to the treatment. The patient was cured after radiological aspiration of the abscess and antibiotic therapy. The abscess did not recur after one year of follow-up.

\subsection{Case 2}

A 29-year-old diabetic female presented to the surgical outpatient department of our hospital with complaints of a lump in her left breast of past 20 days duration in 2017. The patient was not pregnant or lactating at that time. Local examination revealed a lump $4.5 \times 3.5$ $\mathrm{cm}$ in left breast at 2 o'clock position free from skin and underlying muscle. The overlying skin appeared normal, with no inflammation and was non tender. There was no nipple retraction or discharge from the nipple. Axillary lymph nodes were not palpable. Rest of general physical and systemic examination was normal. Provisional diagnosis of breast lump with a suspicion of malignancy was made and following investigations were advised: routine blood investigation, Chest x-ray, USG of both breasts, and core needle biopsy of the left breast lump. Her routine blood investigations were normal. USG of left breast revealed heterogeneously hypoechoic mass lesion of approximately $3.4 \times 2.4 \mathrm{~cm}$ size in the left breast at 2 o'clock position. Core needle biopsy finding showed plenty of neutrophils and inflammatory cells with necrotic background suggestive of breast abscess. Lump excision with radical duct excision was done under sterile conditions. The patient was empirically started on tablet erythromycin $500 \mathrm{mg} 6$ hourly and metronidazole $400 \mathrm{mg} \mathrm{BD}$ for two weeks. The drained pus was collected and sent for microbiological investigations. Gram stain of the drained pus revealed gram-negative bacilli. The culture on blood agar grew grey-white opaque, non-hemolytic colonies and non lactose fermenting colonies on MacConkey's agar. This non-lactose fermentating gram negative bacillus was identified as Salmonella paratyphi A by MALDITOF mass spectrometry using the bioMérieux VITEK MS system (IVD database version 2.0). The antibiotic susceptibility was determined by Kirby Bauer disk diffusion method in accordance with CLSI guidelines and the isolate was susceptible to ciprofloxacin, trimethoprim-sulfamethoxazole, erythromycin, azithromycin, chloramphenicol, ceftazidime, and ceftriaxone ampicillin and nalidixic acid. On receipt of the pus culture report blood culture and widal test were advised. However, both these tests were negative. She was treated with oral ciprofloxacin $500 \mathrm{mg}$ twice daily for 14 days. USG of breast done after two weeks of antibiotic therapy revealed no significant change in the heterogenoushypoechoic lesion. Left breast lumpectomy with left radical milk duct excision was done. Some pus like material was encountered which was sent to microbiology laboratory for culture and 
Table 2. Showing the reports of breast abscess cases due to Salmonella Typhi and Paratyphi infections

\begin{tabular}{|c|c|c|c|c|c|c|}
\hline Year & $\begin{array}{l}\text { Age } \\
\text { of Patient }\end{array}$ & $\begin{array}{l}\text { Underlying } \\
\text { condition }\end{array}$ & $\begin{array}{l}\text { Salmonella } \\
\text { Species }\end{array}$ & $\begin{array}{l}\text { Unilateral/bilateral } \\
\text { breast abscess }\end{array}$ & $\begin{array}{l}\text { Clinical } \\
\text { presentation }\end{array}$ & References \\
\hline 2016 & 60 & Diabetes mellitus & S. Typhi & Unilateral & Acute & Murugesan et al.(10) \\
\hline 2016 & unknown & Unknown & S. Typhi & Unknown & Unknown & Elumalai et al. (11) \\
\hline 2015 & 37 & Diabetes mellitus & S. paratyphi A & Unilateral & Acute & Sood $(5)$ \\
\hline 2014 & 31 & Unknown & S. paratyphi A & Unilateral & Recurrent & Ghadage et al. (6) \\
\hline 2013 & 40 & Unknown & S. Typhi & Unilateral & Acute & Banu et al. (12) \\
\hline 2012 & 33 & Unknown & S. paratyphi A & Unilateral & Chronic & Siddesh et al. (7) \\
\hline 2012 & 60 & Diabetes mellitus & S. Typhi & Unilateral & Acute & Kumar et al. (13) \\
\hline 2012 & 33 & Unknown & S. paratyphi A & Unilateral & Recurrent & Fernando et al. (8) \\
\hline 2011 & 28 & Unknown & S. Typhi & Unilateral & Acute & Vattipally et al. (14) \\
\hline 2011 & 29 & Unknown & S. Typhi & Bilateral & Acute & Singh et al. (15) \\
\hline 2009 & 35 & Unknown & S. Typhi & Bilateral & Acute & Singh et al. (16) \\
\hline 2007 & Unknown & Immunocompromised & S. Typhi & Unilatearal & Acute & Mahajan et al. (17) \\
\hline 2007 & 54 & Unknown & S. Typhi & Unilatearal & Acute & Delori et al. (18) \\
\hline 2003 & 40 & Fibroadenoma of breast & S. Typhi & Unilateral & Acute & Jayakumar et al. (19) \\
\hline 1994 & Unknown & Unknown & S. Typhi & Unknown & Unknown & Lalitha et al. (20) \\
\hline 1972 & 43 & Unknown & S. Typhi & Unilateral & Chronic & Barrett et al. (21) \\
\hline 1907 & 16 & Unknown & S. Typhi & Unilateral & Chronic & Thayer et al. (22) \\
\hline
\end{tabular}

Table 3. Showing the reports of breast abscess cases due to Salmonella Paratyphi infections (5-8)

\begin{tabular}{lllll}
\hline Year & Underlying condition & Unilateral/bilateral breast abscess & Clinical presentation & Treatment \\
\hline 2015 & Diabetes mellitus & Unilateral & Acute & Injectable Ceftriaxone/Duration unknown \\
2014 & Unknown & Unilateral & Recurrent & Injectable cefotaxime/Duration unknown \\
2012 & Unknown & Unilateral & Chronic & Injectable Ceftriaxone for 2 weeks \\
2012 & Unknown & Unilateral & Recurrent & Injectable Ceftriaxone for 6 weeks \\
Case 1 & Gastroenteritis & Unilateral & Acute & Oral azithromycin for five days \\
Case 2 & Diabetes mellitus & Unilateral & Acute & Intravenous ceftriaxone for seven days \\
\hline
\end{tabular}

sensitivity. Culture again grew Salmonella paratyphi A with similar antimicrobial susceptibility pattern. According to the susceptibility pattern of the strain, the antibiotic regimen was rationalized intravenous ceftriaxone 2 gm 12 hourly for seven days. On followup there was complete resolution of abscess.

\section{Discussion}

Salmonella paratyphi A causes paratyphoid fever which is characterized by acute onset of fever, abdominal pain, diarrhoea, nausea and vomiting $(3,4)$. Localized disease can occur following both overt and silent bacteremia followed by seeding of bacteria at distant sites (3). Breast abscess due to Salmonella paratyphi is rare complication of enteric fever. It can be attributed mainly to Salmonella typhi (Table 2). Till date, there are only four reports of breast abscess caused by Salmonella paratyphi A (Table 3) (5-8). The first case of breast abscess due to Salmonella paratyphi A was reported in 2012 by Fernando et al. in a young woman from Bangladesh (8).

We could not determine the source of Salmonella Paratyphi A in both cases, however our first patient gave a history of gastro-enteritis. In enteric fever, dissemination to multiple organ systems following bacteremia may lead to localized abscess formation. Although hematogenous dissemination of Salmonella is a well established and the most likely mechanism of breast abscess, in the second case, this route of dissemination seemed unlikely. However, since our patient is diabetic, we speculate that impairment of cellmediated immunity in diabetes may have impaired the ability of macrophages to kill intracellular pathogens such as Salmonella. Underlying condition was unknown in previously reported Salmonella Paratyphi A cases (5-8). Our both cases had unilateral breast abscess like other reported cases $(5-8)$.

There are cases of recurrent breast abscess caused by Salmonella Paratyphi A reported in literature $(6,8)$. However, unlike all these cases of Salmonella Paratyphi A breast abscess in our patient did not have any recurrence or chronicity of abscess. Kumar reported a multidrug resistant typhoid with breast abscess (9). In the present case, the Salmonella Paratyphi A isolate showed good susceptibility to all the drugs. However, blood culture and widal test results were negative in these patients. But the pus from breast aspirated under sonographic guidance grew the same isolate as pure growth implicating Salmonella Paratyphi A as the causative agent of breast abscess in this patient. The histological examination was also suggestive of breast abscess.

Due to its diverse aetiologies, it can pose a diagnostic challenge and warrants detailed evaluation. As observed in both our cases, the importance of identifying atypical 
agents is that appropriate antimicrobial therapy can be instituted early.

Several line of evidence suggest that Salmonella Paratyphi A isolated from the patients was responsible for the breast abscess: gram stain of aspirated pus was positive for gram negative bacteria, culture of aspirated pus grew Salmonella Paratyphi A, direct evidence of infection was present, there was an absence of other pathogen and infection responded to treatment.

\section{Conclusion}

Appropriate sampling, surgery supplemented by a comprehensive microbiological work up aided in pathogen identification and appropriate antibiotic administration for a successful outcome of these patients.

\section{References}

1. Hohmann EL. Non-typhoidal salmonellosis. Clin Infect Dis. 2001; 32:263-269.

2. BaranI, Aksu N, AksoyA. Breast abscess dueto Salmonella Typhi murium in a patient with rheumatoid arthritis: A casereport. BMC Infect Dis. 2016; 16:348.

3. Cheng SP, Liu CL, Chang YC. Extraintestinal Salmonella infection appearing as a suspicious breast mass. J Ultrasound Med. 2006; 25:277-279.

4. Huang DB, DuPont HL. Problem pathogens: Extraintestinal complications of Salmonella enterica serotype Typhi infection. Lancet Infect Dis. 2005; 5:341-348.

5. Sood S. Breast abscess by Salmonella Paratyphi A: Case report and literature review. J Clin Diagn Res. 2015; 9:DD03-04.

6. Ghadage DP, Wankhade AB, Mali RJ, Bhore AV. Recurrent breast abscess due to Salmonella Paratyphi A: An unusual case. Int J Res Med Sci. 2014; 2:1236-1238.

7. Siddesh G, Sumana MN. A case of breast abscess due to Salmonella Paratyphi A. Int J Health Allied Sci. 2012; 1:109-111.

8. Fernando S, Molland JG, Gottlieb T. Failure of oral antibiotic therapy, including azithromycin, in the treatment of a recurrent breast abscess caused by Salmonella enterica serotype Paratyphi A. Pathog Glob
Health. 2012; 106:366-369.

9. Kumar PD. Breast abscess: A rare complication of multiresistant typhoid fever. Trop Doct. 1998; 4:238-239.

10. Murugesan N, Alagar samy R, Viswanathan MS, Anbazhagan A. A rare case of breast abscess due to Salmonella Typhi. IJCRR. 2016; 8:10-12.

11. Elumalai S, Seetharaman S. Molecular analysis of fluoroquinolone resistance in Salmonella enterica serovar Typhi from a breast abscess case. Indian J Pathol Microbiol. 2016; 59:261.

12. Banu A, Hassan MM, Anand M. Breast abscess: Sole manifestation of Salmonella typhi infection. Indian J Med Microbiol. 2013; 31:94-95.

13. Kumar ES, Selvam RE, Ramesh SS. Salmonella enterica serovar Typhi in breast abscess: A case report. Indian J Pathol Microbiol. 2012; 55:608-609.

14. Vattipally V, Thatigotla B, Nagpal K, Saraiya R, Henry M, Shah AK, Cosgrove J. Salmonella typhi breast abscess: An uncommon manifestation of an uncommon disease in the United States. Am Surg. 2011; 77:E133-135.

15. Singh G, Dasgupta M, Gautam V, Behera A, Ray P. Bilateral breast abscesses due to Salmonella Enterica Serotype Typhi. J Glob Infect Dis. 2011; 3:402-404.

16. Singh S, Pandya Y, Rathod J, Trivedi S. Bilateral breast abscess: A rare complication of enteric fever. Indian $\mathrm{J}$ Med Microbiol. 2009; 27:69-70.

17. Mahajan RK, Duggal S, Chande DS, Duggal N, Hans C, Chaudhry R. Salmonella enterica serotype Typhi from a case of breast abscess. J Commun Dis. 2007; 39:201204.

18. Delori M, Abgueguen P, Chennebault JM, Pichard E, Fanello S. Breast abscess with Salmonella typhi and review of the literature. J Gynecol Obstet Biol Reprod (Paris). 2007; 36:709-712.

19. Jayakumar K, Appalaraju B, Govindan VK. An atypical presentation of Salmonella typhi-A case report. Indian J Med Microbiol. 2003; 21:211-212.

20. Lalitha MK, John R. Unusual manifestations of salmonellosis - A surgical problem. Q J Med. 1994; 87:301-309.

21. Barrett GS, MacDermot J. Breast abscess: A rare presentation of typhoid. Br Med J. 1972; 2:628-629

22. Thayer WS, Hazen HH. Calcification of the breast following a typhoid abscess. J Exp Med. 1907; 9:1-17

(Received April 4, 2018; Revised May 14, 2018 ; Accepted May 16, 2018) 